\title{
A Features Fusion Approach for Neonatal and Pediatrics Brain Tumor Image Analysis Using Genetic and Deep Learning Techniques
}

\author{
https://doi.org/10.3991/ijoe.v17i11.25193 \\ Prashantha S. J. $\left.{ }^{\bowtie}\right)$, H. N. Prakash \\ Visvesvaraya Technological University, Belagavi, India \\ prasi.sjpegmail.com
}

\begin{abstract}
Nowadays, Deep learning (DL) is the growing trend towards creating visual representations of human body organs for clinical analysis, medical interventions as well as to diagnose and treat diseases. This paper propose a method for neonatal and pediatric brain tumors image analysis and prerequisites a T2- weighted MR images only. The pipeline stages of the proposed work as follows: In the first stage, designed a set of specific feature vectors description for high-level classification task using Conventional and deep learning (DL) Feature Extraction methods. The second stage, select a deep features based on proposed convolutional neural network $(\mathrm{CNN})$ method and conventional subset features are from Genetic Algorithm (GA). The third stage, merge the selected features by adapting fusion technique. Finally, predict the brain image is either normal or abnormal. The results demonstrated that the proposed method obtained accurate classification and revealed its robustness to difference in ages and acquisition protocols. The obtained results shows that based on combined deep learning features (DLF) and conventional features have been significantly improves the classification accuracy of the support vector machines (SVM) classifier up to $97.00 \%$.
\end{abstract}

Keywords — conventional features, deep learning features, genetic algorithm, feature fusion, classification

\section{Introduction}

Medical imaging is the process of creating visual representations of the body. The human brain is one of the unique and largest complex organs in the central nervous system (CNS). Medical imaging contributes to an anatomy database representing internal and external structures of the body, making it easier to identify abnormalities of the human brain. According to world health organization (WHO), one -in -six deaths globally due to cancer and estimated 9.6 million deaths in 2018. More than $80 \%$ of the 200,000 new childhood cancers occur annually in developing world [1]. Brain cancer or tumor is one of the serious diseases in the life of human brain development and it is an abnormal growth of cells in the brain. There are several unlike or dissimilar brains 
anatomical and mechanical functioning are present in neonatal period, infancy, childhood and adult's brain.

Understanding the structure, functions and development of brain in health and diseases represents a fascinating challenge in the modern science and have extends a research gateway to examine both abnormal and normal early brain development. Hence, the early period attention has to be paid more for this crucial period. The review and analysis of neonatal to adults' brain MRI is of great interest due to its inherent growth patterns and morphological reshape in Central Nervous System (CNS) disorders. Currently, neonatal to pediatric brain scans of clinical features interpretation is the responsibility of neuroradiologist by using computers to enhance the visualization. This paper presents an effectiveness of high-level tasks with a good feature extraction and makes combinational features accomplishing from neonatal to pediatric brain images. Developing automatic computational approaches for medical image analysis are especially useful in diseases to decide the order of treatment, where patient image must be effectively analyzed and it is essential in clinical trial. Hence, we propose a new automatic intelligent classification model for neonatal to pediatric brain MR image analysis.

The contributions of this paper are $4-$ fold.

- The proposed framework is to design a set of specific feature vectors description for high-level classification task using Conventional and deep learning (DL) Feature Extraction methods.

- Optimal subset significant conventional features obtained from Genetic Algorithm (GA) and deep features are selected by proposed CNN method.

- The proposed model have been adapted the fusion technique to merge the prominent conventional and deep features vectors together to generates a unique fusion features.

- Our proposed model algorithm allows detecting the normal or abnormal of the MRI brains images, with good classification accuracy.

The paper is organized as follows: In Section 2, we describe the related works of traditional feature selection and extraction, deep learning approaches of feature selection and framework of feature level fusion methods. Section 3, Outlines the proposed framework methodology. Features extraction, selection of features vectors, features fusion process and classification are described in Section 4. Section 5, presents experimental setup, the results and a discussion of the methods applied here. Conclusions are drawn in Section 6.

\section{Related work}

From past ten years the researchers have proposed several neonatal and adults brain magnetic resonance imaging (MRI) segmentation, detection and classification methods; but there are notable differences of datasets used, segmentation targets, characteristics and recognition. There is a substantial set of literature on medical image analysis and for designing a type of differentiated features for high - level tasks such as classification and segmentation in the field of medical imaging [2]. Due to subjective nature 
of images many of the previous works focus on feature extraction design and selection approaches. The conventional feature extraction methods include support vector machines [5], feature descriptors [6], random forests [4], color and texture [3].

In recent years, research that seeks to gain insights into deep learning models like feature representation in Convolution Neural Networks (CNNs) [7]. Recently, many research articles have been covered with deep learning approaches such as classification, segmentation, detection and registration [8]. Furthermore, research studies [9]] [10] involve classifying medical images based on deep learning models. Deep learning methods [11] are proposed and applied to medical images for the extraction of features characteristics. There are many researchers proposed several approaches for feature selection such as Deep Neural Networks (DNNs) [13], neural networks [12], Genetic algorithms (GAs) [14] and radial basis function networks [15].

In spite of this, the analysis of texture is adapted in several applications such as document image analysis, face recognition and biomedical image field. In [16] made a comprehensive review of the texture feature extraction methods like statistical modes, structural methods, transform - based procedures etc. In addition, researchers have developed several methods and gainful studies for an extended period in texture analysis [17] for automatic classification of brain tumors image analysis. To impose texture features on images, wavelets [18], gray level co-occurrence matrix (GLCM) features [19] and local binary patterns [20] methods have been used. Haralick et al. [21] proposed a method to estimate the spatial relationship between adjacent pixels in an image using gray-level co-occurrence matrix (GLCM).

The feature fusion is the process of combining different types of feature data together into a single procedure to generate a single structure model of the fusion process. Many research papers have been reviewed on feature data fusion for image classification tasks [22]. Feature fusion data can be performed in distributed or centralized processes and integration (hybrid) of both pre-processed and raw feature data, could also be considered [23].

Recent work [25] has shown that classification of human brain in magnetic resonance (MR) images is possible using two-tier classifier with adaptive segmentation technique, [26] has presents via neural network and the central moments. In [27] proposed the classification of MRI images under two categories, either normal or abnormal based on wavelet-Entropy and Naive Bayes Classifier. Fully automatic diseased and normal human brain classification which is a great importance for research studies.

Furthermore, unlike aforementioned research work on brain image classification, a method can be applied to the classification of images of developing neonates at different ages, as well as young adults. This demonstrates that the proposed method can be applied to the classification tasks based on coronal, sagittal and axial T2 - weighted training images. Hence, we propose a classification approach for neonatal to pediatric brain MR image analysis. 


\section{$3 \quad$ Proposed methodology}

In this section, we describe the framework of the proposed methodology is shown in Figure 1. Our main objective task is to predict whether an MRI brain image is normal or abnormal. The plan of proposed methodology process as follows: 1) to perform skull elimination in the brain input images.2) to perform image enhancement to modify the attributes of an image to make more suitable for a given task. 3) Extract good features vectors by conventional feature extraction methods and deep learning features vectors by CNN model. 4) Obtaining an optimal conventional feature subset using GA. In parallel, select a set of significant deep features by proposed CNN method. 5) To adopt a features level fusion process to make the discriminatory functional features for classification. 6) To classify images into normal or abnormal using classifier. The comprehensive objective of the proposed framework is to study the MRI brain scans, adopt a features fusion process and make high-level classification task by combining conventional feature extraction and deep learning features (DLF).

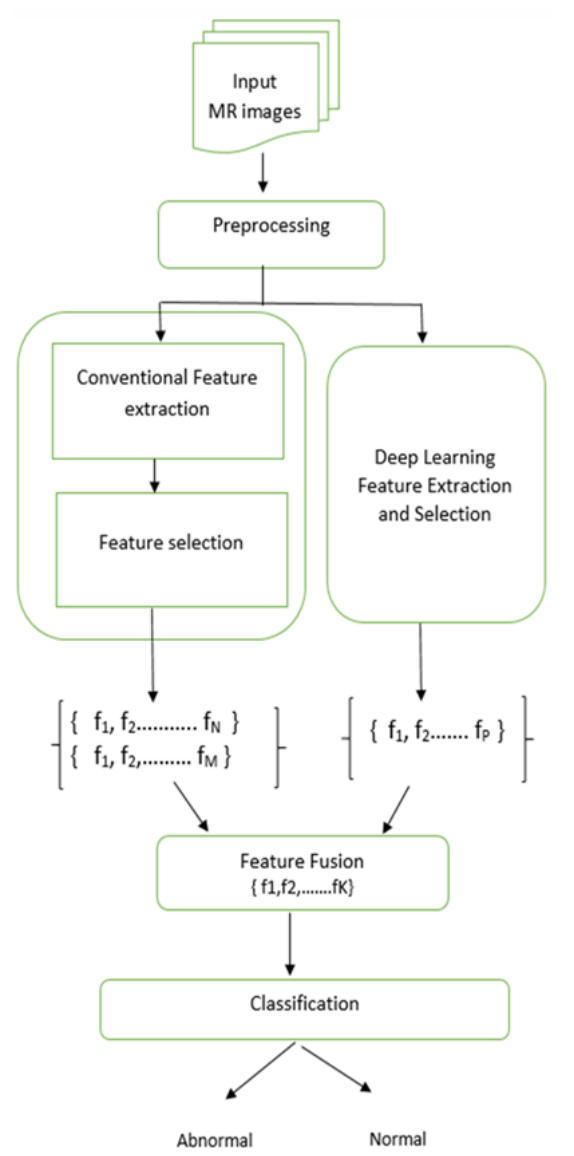

Fig. 1. Framework of the Proposed Architecture 


\subsection{Dataset}

The dataset consists of 41 different subjects/cases T2 weighted Axial, Coronal and Sagittal MRI images are collected from radiopaedia.org online database. The resources of dataset have been primarily compiled across the world by radiologists. The subject's age ranged from the day on birth to 10 years old. The image resolution size is varied from 256x256 to $1024 \times 1024$ pixels. Table 1 provides basic details of the dataset.

Table 1. Acquisition parameters for the MR images

\begin{tabular}{|l|c|}
\hline \multicolumn{1}{|c|}{ Parameters } & MRI Subjects/Cases \\
\hline Number of Subjects & 41 \\
\hline Age & 0 to 10 years \\
\hline Acquistion protocol & $\begin{array}{c}\text { Coronal T2 weighted. } \\
\text { Axial T2 weighted. } \\
\text { Sagittal T2 weighted }\end{array}$ \\
\hline Number of Image slices & 100 \\
\hline Reconstruction Matrix & $256 \times 256 \times 100$ \\
\hline
\end{tabular}

\subsection{Preprocessing}

Preprocessing is an important process and necessary stage to promise the performance of the classification method. We have employed the pre-processing steps involving gray scale image conversion, image resizing (conversion to 256x256 pixel size) using bicubic method, skull stripping and contrast enhancement. In the proposed research work, Contrast Limited Adaptive Histogram Equalization (CLAHE) method [24] is used to improve the contrast of MRI slice images.

\subsection{Feature extraction and selection}

Conventional feature extraction and selection. The present section, describes the proposed conventional strategies of texture features extraction and selection process. In this proposed work, grey level co-occurrence matrix (GLCM) and Haralick textures patterns of an image are computed based on image directions or orientations [17] [21]. The Table 2 shows the details of the proposed texture features. The GLCM matrix reveals the certain properties of the spatial relationships of the gray levels in the image. The Haralick features are computed from the co-occurrence matrix to describing the textures of an image. The image pixels are located within the region of interest (ROI) and considered the relationship of discretized intensities of neighbouring pixels are distributed in one of the image orientations. 
Paper-A Features Fusion Approach for Neonatal and Pediatrics Brain Tumor Image Analysis Using...

Table 2. GLCM and Haralick texture features

\begin{tabular}{|c|c|c|c|c|c|}
\hline \multicolumn{4}{|c|}{ GLCM Texture features } & \multicolumn{2}{|c|}{ Haralick texture features } \\
\hline $\begin{array}{c}\text { Feature } \\
I D\end{array}$ & $\begin{array}{c}\text { Feature } \\
\text { Description }\end{array}$ & $\begin{array}{c}\text { Feature } \\
\text { ID }\end{array}$ & $\begin{array}{c}\text { Feature } \\
\text { Description }\end{array}$ & Feature ID & Feature Description \\
\hline $\mathrm{F} 1$ & $\begin{array}{l}\text { Maximum } \\
\text { probability }\end{array}$ & F13 & Dissimilarity & F1 & Energy \\
\hline F2 & Average & F14 & Inverse Difference & $\mathrm{F} 2$ & Contrast \\
\hline F3 & variance & F15 & $\begin{array}{l}\text { Normalized inverse } \\
\text { Difference }\end{array}$ & F3 & Correlation \\
\hline F4 & entropy & F16 & $\begin{array}{l}\text { Inverse Difference } \\
\text { Moment }\end{array}$ & F4 & Sum of Variances \\
\hline F5 & $\begin{array}{l}\text { Difference } \\
\text { average }\end{array}$ & F17 & $\begin{array}{l}\text { Normalized inverse } \\
\text { Difference moment }\end{array}$ & F5 & Inverse Difference Moment \\
\hline F6 & $\begin{array}{l}\text { Difference } \\
\text { variance }\end{array}$ & F18 & Inverse variance & F6 & Sum Average \\
\hline F7 & $\begin{array}{l}\text { Difference } \\
\text { entropy }\end{array}$ & F19 & Correlation & F7 & Sum Variance \\
\hline F8 & Sum average & F20 & Autocorrelation & F8 & Sum Entropy \\
\hline F9 & Sum variance & F21 & Cluster tendency & F9 & Entropy \\
\hline F10 & Sum entropy & F22 & Cluster shade & F10 & Difference Variance \\
\hline F11 & $\begin{array}{l}\text { Angular second } \\
\text { moment }\end{array}$ & $\mathrm{F} 23$ & Cluster prominence & F11 & Difference Entropy \\
\hline & & & & F12 & $\begin{array}{c}\text { Information Measures of } \\
\text { Correlation } 1\end{array}$ \\
\hline
\end{tabular}

We have adopted a Genetic Algorithm Feature Selection (GAFS) wrapper model, which has been used to select prominent features. The feature subset selection process of the GAFS wrapper model is shown in Figure 2. It involves three subsequent steps. In the first step, subsets of features have been discovered. Second, to determine the significance of the selected subset of features as a result of performance of the classifier. Finally, repeat the first and second steps until the desired iteration is achieved. One of the main objectives of this work is to select a subset of significant features by GAFS module. 


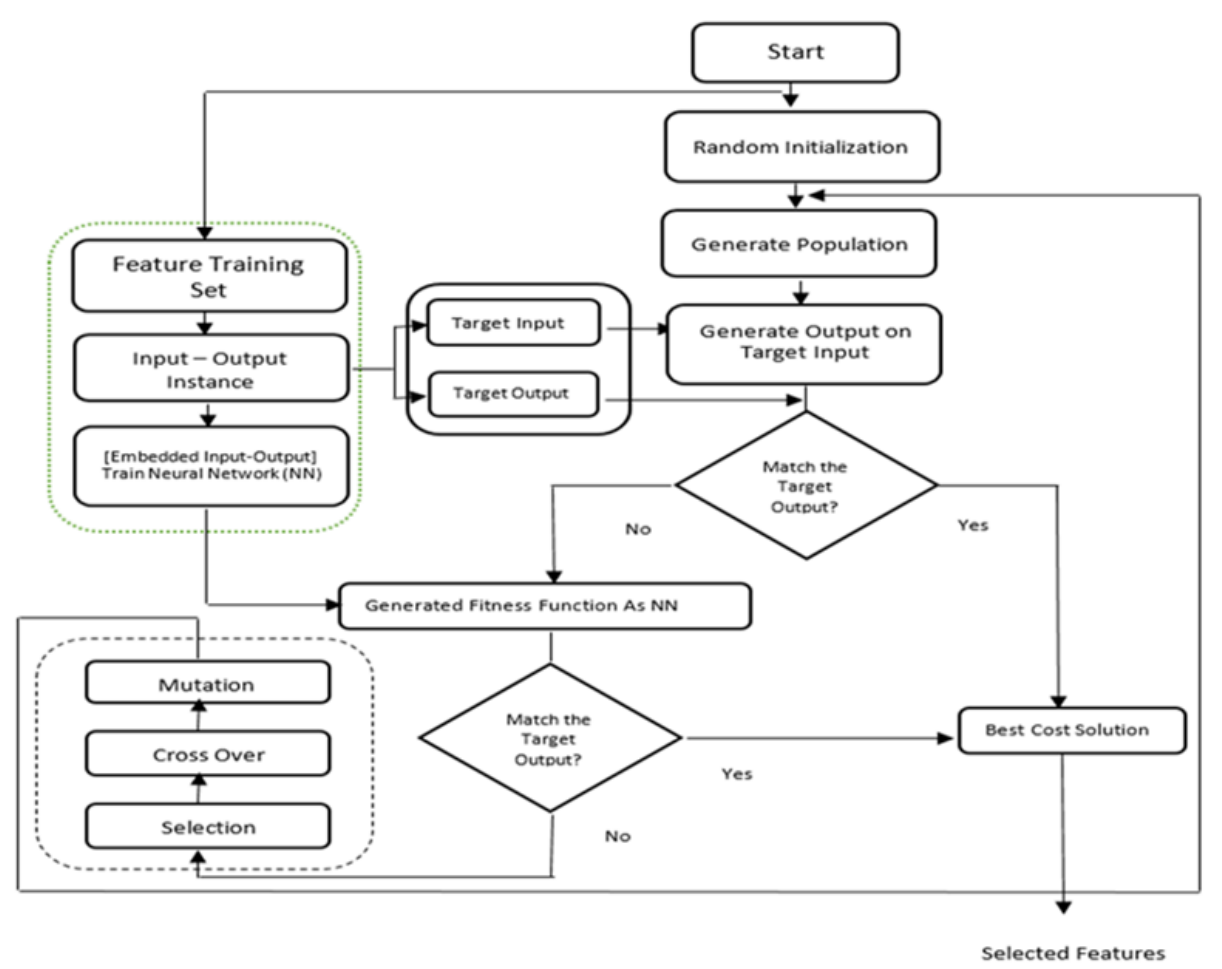

Fig. 2. Work flow of Genetic Algorithm Feature selection (GAFS) wrapper model

Deep learning feature extraction and selection. We initiate a convolutional neural network $(\mathrm{CNN})$-based feature extraction strategy for extracting the ingrained features. The proposed $\mathrm{CNN}$ architecture consists of an input layer followed by two convolutional layers and two subsampling layers, ending with two fully connected layers. The Figure 3 shows the deep feature extraction and selection approach. The input to a convolutional layer is a $\mathrm{Z} \times \mathrm{Z} \times \mathrm{P}$ image where $\mathrm{Z}$ is the height and width of the image and $\mathrm{P}$ is the number of images. The convolutional layer will have $\mathrm{N}$ filters or kernels of size $\mathrm{M} \times \mathrm{M} \times \mathrm{Q}$ where $\mathrm{M}$ is smaller than the dimension of the image and Q can either be the same as the number of channels or smaller and may vary for each kernel. The size of the filters gives rise to the locally connected structure which is convolved each time with the image to produce $\mathrm{N}$ feature maps of size $\mathrm{Z}-\mathrm{M}+1$. The main idea behind the proposed feature extraction and selection approach is to investigate the contribution of each of the input dimensions and inputs of the feature maps of each layer. The Table 3 shows the outline of the CNN architecture which is used in this study. 


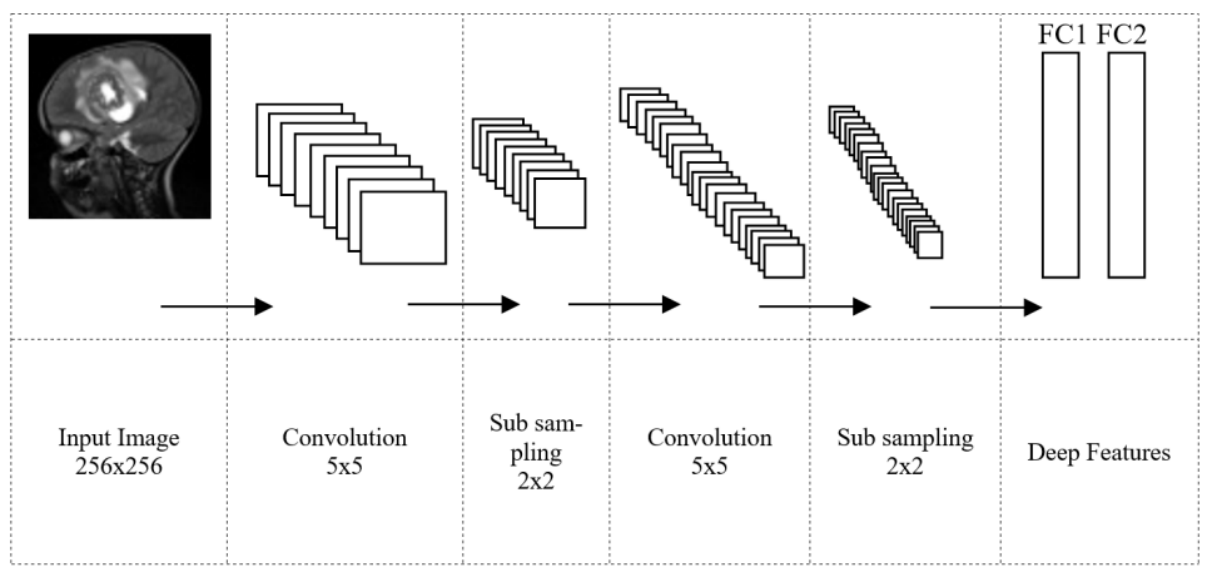

Fig. 3. Proposed deep feature extraction and selection of the CNN framework

Table 3. The details of the proposed $\mathrm{CNN}$ architecture

\begin{tabular}{|l|l|c|c|c|}
\hline Layer & \multicolumn{1}{|c|}{ Layer Type } & size & $\begin{array}{c}\text { Feature Map/output } \\
\text { shape }\end{array}$ & $\begin{array}{c}\text { Selected Feature } \\
\text { maps/neurons }\end{array}$ \\
\hline 1 & Input Layer & $256 \times 256$ & - & 65536 \\
\hline 2 & Convolution + Sigmoid & $5 \times 5 \times 9$ & $252 \times 252 \times 9$ & 571536 \\
\hline 2 & Sub sampling layer & $2 \times 2 \times 9$ & $126 \times 126 \times 9$ & 142884 \\
\hline 3 & Convolution + Sigmoid & $5 \times 5 \times 135$ & $122 \times 122 \times 135$ & 2009340 \\
\hline 3 & Sub sampling layer & $2 \times 2 \times 15$ & $61 \times 61 \times 15$ & 55815 \\
\hline 4 & Fully connected-1(FC 1) layer & $1 \times 3000$ & 3000 & 3000 \\
\hline 4 & Fully connected-2 (FC 2) layer & $1 \times 300$ & 300 & 300 \\
\hline
\end{tabular}

Features fusion process. Fusion is one of the important processes to make the most efficient features set in this proposed methodology. In the proposed work, we have considered fusion process at feature level. It assists us to classify the objects as normal or abnormal by obtaining a set of fused features.

Let $\{\mathrm{Xi} \ldots \mathrm{n}, \mathrm{Yi} \ldots . . \mathrm{m}\}$ be the feature vectors acquired at two different sources. Here $\mathrm{X}$ and $\mathrm{Y}$ represent the feature vectors of conventional features and deep features respectively. The corresponding fused feature vectors denoted as $\{\mathrm{Zi} \ldots \ldots . \mathrm{k}\}$. The identification of a compact set of salient features from feature-level fusion that can enhance recognition accuracy. After extracting the high-level deep features and conventional features, we adapted a linear feature fusion approach to fuse the features i.e., which is to set a fixed proportion $\beta$, as the feature fusion process. The fusion features for classification are computed as follows:

$$
\mathrm{NFV}=\beta . \mathrm{X}+(1-\beta) . \mathrm{Y}
$$

Where NFV is the fusion feature vectors, $\mathrm{X}$ is the vectors of conventional features, and $\mathrm{Y}$ is the vectors of deep features. The $\beta$ is the weight parameter that represent the 
importance between two different features. The fusion features will passed to the classifier for the final classification task.

Classification. In this work, we adopted the support vector machine (SVM) classifier for the purpose of classification. The SVM is a binary classification method and it involves two steps namely training and testing. In the training step, feeding known data to the support vector machine along with previously known decision values to form a finite training set. From this training set, SVM gets its intelligence to classify unknown data. The class label of training images is assigned manually and SVM used for this labeled data. Further, the testing step involved predicting the model results and a set of observations used to determine the performance of the model. In SVM classifier, the features of a test brain MR image is match-up the features of all trained set images. So, the proposed approach is applied to brain MR images in order to classify either as a normal or abnormal using support vector machine.

\section{Experimentation}

\subsection{Experimental setup}

In this section, we have presented the details of experimentation conducted on the publicly available radiopaedia.org online database along with the obtained results. This dataset has categorized into two classes i.e., normal or abnormal. The Figure 4 shows the pre-processing results of typical images. The number of input dataset is 100 out of which 50 are of the normal brain and 50 are of abnormal brain. The GLCM, Haralick textures and deep learning features are extracted in the feature extraction stage. In the feature selection stage, conventional features are selected by genetic algorithm and deep features are selected by proposed CNN (9c-2s-15c-2s-2FC) approach. Further, after extracting the prominent deep features and conventional features, we have adapted a linear feature fusion approach to fuse the features and have used for classification task. In the experiment, GLCM + Harlick (C1), Fusion features of GLCM + Harlick (C2), Fusion features of GLCM + Harlick + DL FC-2 (C3) along with three individual features (GLCM, Haralick, DL FC-1 \&FC-2) are considered separately. In the experimental study, a total of 148 conventional features are extracted, including 96 GLCM features and 52 Harlick features. The Figure 5a. shows the number of selected features using GAFS wrapper method. In our proposed classification system, two sets of images are taken to check the working of the classifier. The first set, consists of randomly selected the images which are already used for training and second set consists of all 100 images which are classified by our proposed system. The performance of the system is evaluated from confusion matrix and results are discussed in the section 4.2. 


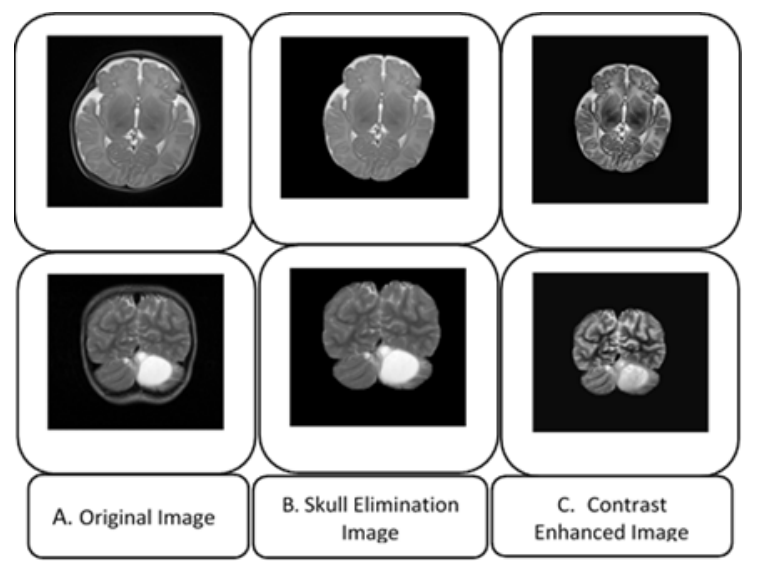

Fig. 4. Examples of (A) original Image (B) Skull elimination (C) Contrast Enhancement MR Images

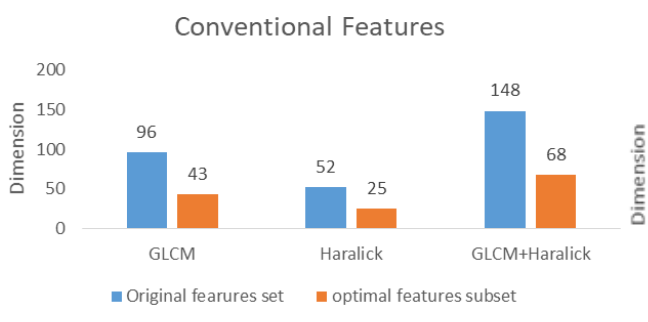

(a)

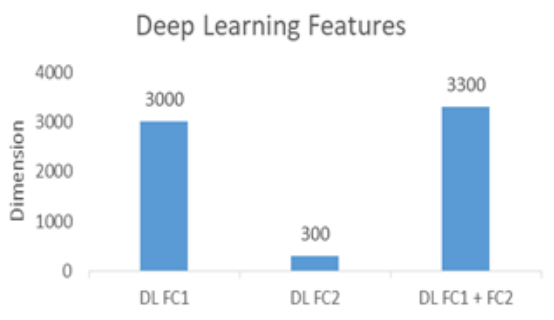

(b)

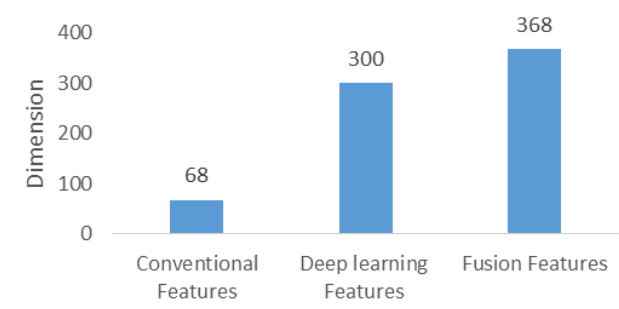

(c)

Fig. 5. (a-c) Features description of the proposed method.

\section{$5 \quad$ Results and discussion}

In this section, we will show the experimental results and examine the performance of the proposed classifiers. In the experiments of GAFS model, genetic algorithm is extensively used for feature selection and outcome is strongly dependent on population diversity and selective pressure. The table 4 shows setting of the parameters based on 
the outcomes of several prior runs by the genetic algorithm. In this study fitness proportionate selection strategy is applied and the probability of selection of the highest probability individual is $\mathrm{p}$ has computed by equation (2).

$$
p_{i}=\frac{f_{i}}{\sum_{j}^{N} f_{j}}
$$

If $f_{i}$ is the fitness of individual $i$ in the population, its probability of being selected by eq 2 and where $N$ is the number of individuals in the population. The fitness of an individual is determined by evaluating the neural network constructed in GAFS method and outputs an optimal subset of features. The Table 4 shows the parameters of genetic algorithm based on prior runs.

Table 4. Genetic Algorithm Parameters.

\begin{tabular}{|l|c|}
\hline \multicolumn{1}{|c|}{ Parameter } & Value \\
\hline Maximum Number of Iterations & 10 \\
\hline Population Size & 10 \\
\hline Crossover Percentage & 0.7000 \\
\hline Number of Off springs (Parents) & 8 \\
\hline Mutation Percentage & 0.3000 \\
\hline Parameter & Value \\
\hline Maximum Number of Iterations & 10 \\
\hline
\end{tabular}

In this experimentation study, we investigate $\mathrm{CNN}$ architecture, composed of 9 convolution layers with $5 \times 5$ kernel size, sub sampling layer $2 \times 2,15$ convolution layer, sub sampling layer $2 \times 2$ and two fully connected (FC 1 and FC 2) layers are added to get the deep features. The following hyper parameters are used to configure the network as follows: Learning Rate is 0.001 ; Number of epochs is 50; Batch size is 100; Activation function is sigmoid; Weight Initialization is randomly. The parameters are setting based on results of several prior experiments. The convolution responses of the acquired images are shown in the Figure 6.

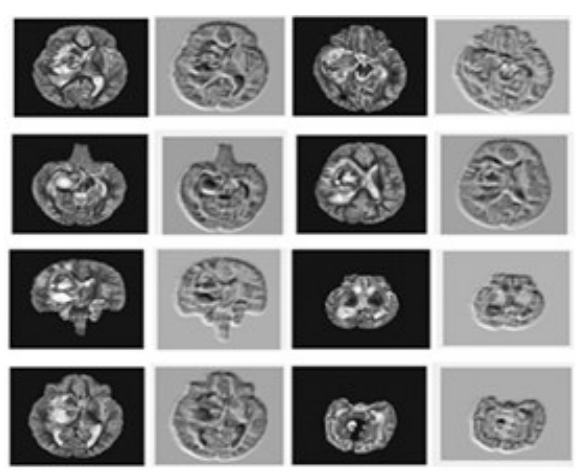

Fig. 6. Trained convolution images in the first layer after 20 epochs (From left to Right: T2weighted train image, Convolved Imag.) 
At the end of feature selection process, we have obtained 68 optimal feature subset from the original conventional features group. In addition to that, 3000 and 300 deep features are obtained from first fully connected layer FC1 and second fully connected layer FC2 respectively. The Figure $5 \mathrm{~b}$. Shows the number of selected features using deep leaning. The present feature fusion work includes a linear feature fusion approach and it produces a new feature vectors. In this stage, 368 dimension of fused features vectors are generated based on 68 conventional features and 300 deep features of second fully connected layer FC2 are selected are shown in Figure 5c. The Figure 7 (a b). shows the obtained mean and standard value of proposed fusion features.

The evaluation of proposed method is achieved by seven different sets of experiments. In this work, all types of feature vectors are used as inputs. The objective of all these experiments is to provide an adequate basis for comparison. In the experimental setup, we have calculated $2 \times 2$ confusion matrix, where 2 is the number of predicted classes. The Table 5 shows the classification confusion matrix for performing the proposed approach. By using conventional texture and deep learning features analysis methods are examine based on confusion matrix. This can be described by the terms as True Positive (TP), True Negative (TN), False Positive (FP) and False Negative (FN) as follows:

- True Positive (TP) - abnormal class images are predicted correctly from the model, - True Negative (TN) - normal class images are predicted correctly from the model,

- False Positive (FP) - normal class images are predicted incorrectly as inclusion to the abnormal class from the model, and

- False Negative (FN) - abnormal class images are predicted incorrectly as inclusion to the normal class from the model.

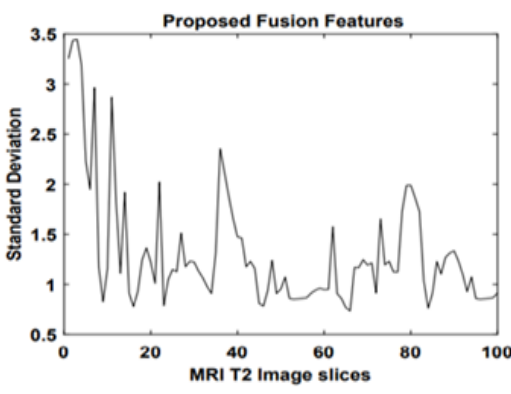

(a)

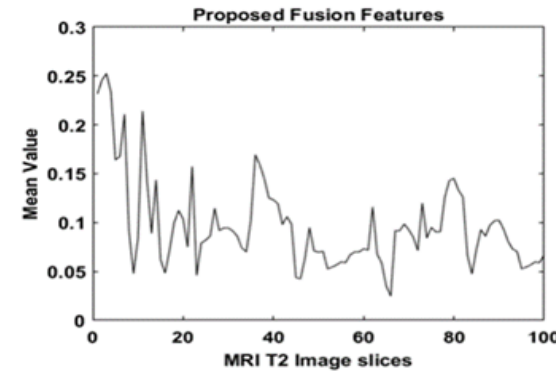

(b)

Fig. 7. (a - b) Mean and Standard value of the proposed fusion features 
Table 5. Classification confusion matrix's for the SVM classifier.

\begin{tabular}{|l|c|c|c|c|}
\hline \multicolumn{1}{|c|}{ Features } & TP & TN & FP & FN \\
\hline GLCM & 33 & 39 & 17 & 11 \\
\hline Haralick & 33 & 47 & 17 & 3 \\
\hline GLCM + Haralick & 33 & 49 & 17 & 1 \\
\hline Fusion features (GLCM + Haralick ) & 41 & 49 & 9 & 1 \\
\hline DL FC-1 & 43 & 49 & 7 & 2 \\
\hline DL FC-2 & 45 & 49 & 5 & 1 \\
\hline Fusion features (GLCM + Haralick + DL FC-2) & 48 & 49 & 2 & 1 \\
\hline
\end{tabular}

The Table 6 shows the comprehensive performance evaluation results by the proposed classification model. We compared the proposed work with other reported recent studies classification accuracy results and demonstrated the robustness of the proposed method shown in Table 7. The experimental results by SVM classification is shown in Figure 8 .

Table 6. Performance of the proposed model.

\begin{tabular}{|l|c|c|c|c|c|c|}
\hline Features & Sensitivity & Specificity & Precision & Recall & F-MEASURE & Accuracy (\%) \\
\hline GLCM & 0.7500 & 0.6964 & 0.6600 & 0.7500 & 0.7021 & $72.00 \%$ \\
\hline Haralick & 0.9167 & 0.7344 & 0.6600 & 0.9167 & 0.7674 & $80.00 \%$ \\
\hline GLCM + Haralick & 0.9706 & 0.7424 & 0.6600 & 0.9706 & 0.7857 & $82.00 \%$ \\
\hline $\begin{array}{l}\text { Fusion features (GLCM } \\
\text { + Haralick ) }\end{array}$ & 0.9762 & 0.8448 & 0.8200 & 0.9762 & 0.8913 & $90.00 \%$ \\
\hline DL FC-1 & 0.9556 & 0.8727 & 0.8600 & 0.9556 & 0.9053 & $91.00 \%$ \\
\hline DL FC-2 & 0.9756 & 0.9074 & 0.9000 & 0.9782 & 0.9053 & $94.00 \%$ \\
\hline $\begin{array}{l}\text { Fusion features (GLCM } \\
\text { + Haralick + DL FC-2) }\end{array}$ & 0.9762 & 0.8448 & 0.8200 & 0.9762 & 0.8913 & $97.00 \%$ \\
\hline
\end{tabular}

Table 7. Comparison of previous studies along with the proposed method.

\begin{tabular}{|l|c|c|}
\hline \multicolumn{1}{|c|}{ Study } & Number of images & Accuracy (\%) \\
\hline$[25]$ & 65 & 94.28 \\
\hline$[26]$ & 60 & 88.33 \\
\hline$[27]$ & 64 & 92.60 \\
\hline Proposed Method & 100 & 97.00 \\
\hline
\end{tabular}




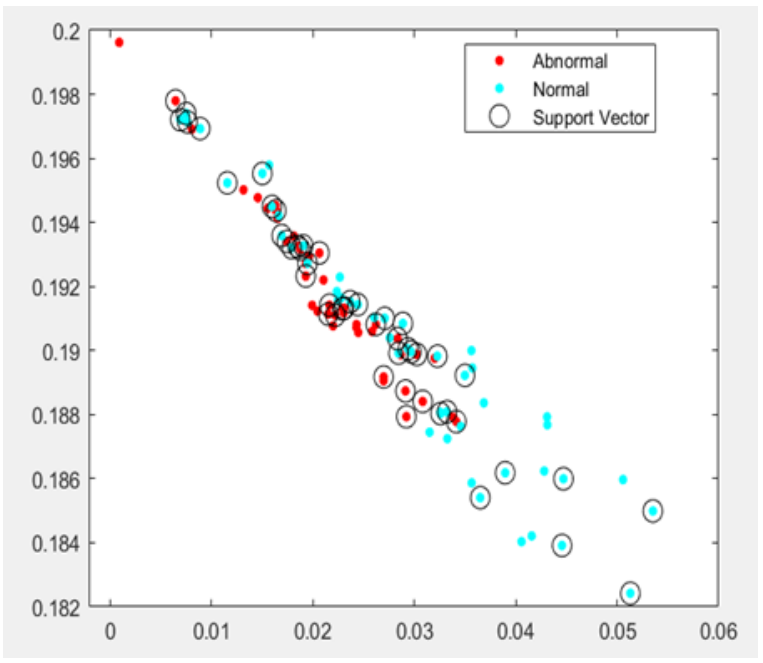

Fig. 8. Experiment results by SVM.

\section{Conclusion}

In this paper, we have presented an automatic classification method of MR brain images and it shows the obtained classification results of images acquired at different ages ( 0 to 10 years). We employed a GAFS wrapper method and proposed CNN architecture to perform the features extraction and selection. Then, we have applied a fusion process to make the complementary fusion features set which is considered and produces a better recognition than individual features set. For each MRI image, 368 subset features are selected in the proposed method. The obtained experimental results show that most promising results. The classification accuracy $97.00 \%$ have been achieved based on the collected T2 - weighted MRI images of the brain.

\section{$7 \quad$ References}

[1] Barr R et al., (2006). "Pediatric Oncology resource", pp. 1605-1617.

[2] C. Barata, M. Ruela, M. Francisco, T. Mendonça, and J. S. Marques, (2014). "Two systems for the detection of melanomas in dermoscopy images using texture and color features," IEEE Systems Journal, vol. 8, no. 3, pp. 965-979. https://doi.org/10.1109/jsyst.2013. 2271540

[3] F. Riaz, A. Hassan, R. Nisar, M. Dinis-Ribeiro, and M. Coimbra, (2017). "Content-adaptive region-based color texture descriptors for medical images," IEEE Journal of Biomedical and Health Informatics, vol. 21, no. 1, pp. 162-171. https://doi.org/10.1109/jbhi.2015.2492464

[4] J. Ramirez, J. M. Gorriz, F. Segovia et al., (2010). "Computer aided diagnosis system for the alzheimer's disease based on partial least squares and random forest spect image classification," Neuroscience Letters, vol. 472, no. 2, pp. 99-103. https://doi.org/10.1016/j.neulet. $\underline{2010.01 .056}$ 
Paper-A Features Fusion Approach for Neonatal and Pediatrics Brain Tumor Image Analysis Using...

[5] Y. Zhang, S. Chen, S. Wang, J. Yang, and P. Phillips, (2015). "Magnetic resonance brain image classification based on weighted-type fractional Fourier transform and nonparallel support vector machine," International Journal of Imaging Systems and Technology, vol. 25 , no. 4, pp. 317-327. https://doi.org/10.1002/ima.22144

[6] D G Lowe, (2004). "Distinctive image features from scale- invariant key points," IJCV, Vol 60, No 2, pp. 91-110.

[7] Ben Athiwaratkun and Keegan Kang, (2015). "Feature Representation in Convolutional Neural Networks", arXiv: 1507.02313v1 [cs.CV] 8 Jul 2015.

[8] Mohammad Hesam Hesamian et.al., (2019). "Deep learning Techniques for medical Image segmentation: Achievements and challenges", Journal of digital imaging, 32:582-596 https://doi.org/10.1007/s10278-019-00227-x

[9] C. N. Vasconcelos and B. N. Vasconcelos, (2017). "Experiments using deep learning for dermoscopy image analysis," Pattern Recognition Letters, https://doi.org/10.1016/j. patrec.2017.11.005

[10] X.Yang and Y Fan, (2018). "Feature extraction using convolutional neural networks for multi-atlas based image segmentation", Proc SPIE Vol 10574, Mar Art no 1057439.

[11] G S.Tandel et. al. (2019). "A review on a deep leaning perspective in brain cancer classification." Cancer, vol 11, no1, pp. 11.

[12] A.Verikas and M.Bacauskiene,(2002). "Feature selection with neural networks", Elsevier Pattern Recognition Letters 23 pp.1323-1335. https://doi.org/10.1016/s0167-8655 (02)00081-8

[13] Roy, D., Murty, K. S. R., \& Mohan, C. K. (2015). Feature selection using Deep Neural Networks. 2015 International Joint Conference on Neural Networks (IJCNN). https://doi. org/10.1109/ijenn.2015.7280626

[14] Fernando Gomez, Alberto Quesada, and Roberto Lopez, Artelnics. 2020. "Genetic algorithms for feature selection”, neuraldesigner.com (C) 2020, Artificial Intelligence Techniques, Ltd.

[15] J. Basak and S. Mitra, (1999). "Feature selection using radial basis function networks," Neural computing \& applications, vol. 8, no. 4, pp. 297-302. https://doi.org/10.1007/s00 $\underline{5210050035}$

[16] Anne Humeau-Heurtier, (2019). "Texture Feature Extrction Methods: A Survey”, IEEE Access, Vol 7. https://doi.org/10.1109/access.2018.2890743

[17] S.Tantisatirapong,(2015). "Texture Analysis of multimodal magnetic resonance images in support of diagnostic classification of Childhood brain tumors", Ph.D., dissertation, School Electron., Elect.Comput.Eng.,Univ.Birmingham, Birmingham,U.K.

[18] Dyck, D. V. (1997). Wavelets for texture analysis, an overview. IET Conference Proceedings 581-585.

[19] Zwanenburg, A., Vallières, M., Abdalah, M. A., Aerts, H. J. W. L., Andrearczyk, V., Apte, A., Boellaard, R. (2020). The Image Biomarker Standardization Initiative: Standardized Quantitative Radiomics for High- Throughput Image-based Phenotyping. Radiology, 191145. https://doi.org/10.1148/radiol.2020191145

[20] Ojala, T., Pietikainen, M. \& Harwood, D. (1994). Performance evaluation of texture measures with classification based on Kullback discrimination of distributions. Pattern Recognition, Vol. 1-Conference A: Computer Vision amp; Image Processing., Proceedings of the 12th IAPR International Conference on 1, 582-585. https://doi.org/10.1109/ icpr.1994.576366

[21] Haralick R. M., Shanmugam K. \& Dinstein I. (1973). Textural Features for Image Classification. IEEE Transactions on Systems, Man, and Cybernetics 3, 610-621 https://doi.org/10. $\underline{1109 / \text { tsmc. } 1973.4309314}$ 
Paper-A Features Fusion Approach for Neonatal and Pediatrics Brain Tumor Image Analysis Using...

[22] Lai, Z., \& Deng, H.(2018), "Medical Image Classification Based on Deep Features Extracted by Deep Model and Statistic Feature Fusion with Multilayer Perceptron. Computational Intelligence and Neuroscience, pp.1-13. https://doi.org/10.1155/2018/2061516

[23] Smolinska, A., Engel, J., Szymanska, E., Buydens, L., \& Blanchet, L. (2019). General Framing of Low-, Mid-, and High-Level Data Fusion with Examples in the Life Sciences. Data Fusion Methodology and Applications, pp.51-79. https://doi.org/10.1016/b978-0-44463984-4.00003-X

[24] K. Zuiderveld.,(1994). “Contrast Limited Adaptive Histogram Equalization” In: P. Heckbert: Graphics Gems IV, Academic Press 1994, ISBN 0-12-336155-9. https://doi.org/10. 1016/b978-0-12-336156-1.50061-6

[25] Anitha, V., \& Murugavalli, S. (2016). Brain tumour classification using two-tier classifier with adaptive segmentation technique. IET Computer Vision, 10(1), 9-17. https://doi.org /10.1049/iet-cvi.2014.0193

[26] Ouchtati, S., Sequeira, J., Aissa, B., Djemili, R., \& Lashab, M. (2018). Brain tumors classification from MR images using a neural network and the central moments. 2018 International Conference on Advanced Systems and Electric Technologies (IC_ASET). https://doi.org/10.1109/aset.2018.8379898

[27] Zhou, X., Wang, S., Xu, W., Ji, G., Phillips, P., Sun, P., \& Zhang, Y. (2015). Detection of Pathological Brain in MRI Scanning Based on Wavelet-Entropy and Naive Bayes Classifier. Lecture Notes in Computer Science, 201-209. https://doi.org/10.1007/978-3-319-16483$\underline{0} 20$

[28] Hamed \&Yaghoub (2020). "Clustering of Brain Tumors in Brain MRI Images based on Extraction of Textural and Statistical Features", International Journal of Online and Biomedical Engineering, vol.16 no.12. pp.116 -131. https://doi.org/10.3991/ijoe.v16i12.16929

[29] M. Samir et al. (2020). "Detection and Classification of White Blood Cells Through Deep Learning Techniques", International Journal of Online and Biomedical Engineering (iJOE), val 16 no 15. pp 94 -105. https://doi.org/10.3991/ijoe.v16i15.15481

[30] Yishui Shui et.al.,(2016). "An Improved Method of Genetic Algorithm to Solve the Variable Speed Limit Problem with Constraint Conditions ", International Journal of Online and Biomedical Engineering(iJOE),vol12 no12 pp.16-22. https://doi.org/10.3991/ijoe.v1 $\underline{2 \mathrm{i} 12.6448}$

\section{Authors}

Prashantha S. J. received degree in Bachelor of Computer Science and Engineering from Visvesvaraya Technological University, Belgaum, Karnataka. India. and M.Tech in Computer Science and Engineering from Visvesvaraya Technological University, Belgaum, Karnataka. India. Currently he is pursuing Ph.D. in Computer Science and Engineering at Visvesvaraya Technological University, Belgaum, Karnataka, India. He is currently working as Assistant Professor in Department of Computer Science and Engineering at Adhichunchanagiri Institute of Technology, Chikkamagaluru, Karnataka, India. He has 15 years of teaching experience. His research interest includes image processing, Bio-Medical Image Analysis, pattern recognition, Machine Learning and Deep Learning. He has published papers in National/ International conferences and Journal. 
Dr. H. N. Prakash received degree in Bachelor of Electronics and Communications and Engineering from Mysore University, Karnataka, India, M.Tech in Electronics Instrumentation from National Institute of Technology Warangal, Telangana, India and Ph.D in Computer Science from Mysore University, Karnataka, India. He is currently working as Professor and Head in Department of Computer Science \& Engineering at Rajeev Institute of Technology, Hassan, Karnataka, India. He has 29 years of teaching experience. His research interest includes image processing, pattern recognition, Artificial Intelligence, Artificial Neural Network and Machine Learning. He has published many papers in National and International conferences and Journals. He has published IEEE transaction in Pattern Recognition and Machine Intelligence.

Article submitted 2021-07-01. Resubmitted 2021-08-04. Final acceptance 2021-08-05. Final version published as submitted by the authors. 